\title{
Revised diagnostic criteria for Marfan syndrome
}

\author{
P Brennan \\ Clinical Director, Northern Genetics Service, Newcastle upon Tyne, UK
}

TITLE The revised Ghent nosology for the Marfan syndrome

\author{
Correspondence to $P$ Brennan, \\ Institute of Human Genetics, \\ International Centre for Life, \\ Central Parkway, \\ Newcastle upon Tyne \\ NEI 3BZ, UK
}

DECLARATION OF INTERESTS No conflicts of interest declared. tel. $+44(0) 1912418500$

e-mail paul.brennan@nuth.nhs.uk

\section{SUMMARY}

In general terms, the diagnosis of Marfan syndrome (MFS) is relatively straightforward in many cases but can be difficult in others, especially children and adolescents. This international consensus statement on the diagnosis of MFS, written by a panel of acknowledged experts from Europe and the US, replaces the 1996 Ghent criteria which formed the basis of the Scottish Intercollegiate Guidelines Network (SIGN) Marfan syndrome guideline.' The new Ghent nosology gives more diagnostic weight to the two key features of MFS, namely aortic root dilatation/aneurysm and ectopia lentis - so that, for example, an individual with both features can be diagnosed with MFS even in the absence of a positive family history or other physical features. Previous diagnostic categories (skeleton, skin, dura, lungs) have been resolved and simplified into a single 'systemic score'. The nosology also creates a more prominent role for mutation analysis of the FBNI gene and other genes known to cause 'MFS-like' syndromes and gives guidance on when to consider and how to investigate - such alternative diagnoses. It offers brief guidance on clinical management, particularly of aortic disease.

\section{OPINION}

As with most genetic disorders, accurate diagnosis of MFS can be challenging for a number of reasons: firstly, MFS evolves with time, so confident diagnosis in the young can be challenging; secondly, people with MFS can manifest with different disease features, even in the same family - although aortic disease and ectopia lentis usually 'breed true'; thirdly, not everyone with MFS manifests a given disease feature completely - some features are mild and subtle, and may be indistinguishable from population variation; fourthly, over the last 20 years, a number of new 'overlap' syndromes have been identified and described - for example, familial thoracic aortic aneurysm/dissection, Loeys-Dietz syndrome, vascular Ehlers-Danlos syndrome; and finally, while FBN I mutation analysis has become increasingly adopted in the UK, it is not $100 \%$ sensitive - so the use of DNA analysis as a primary diagnostic test remains limited in the one patient group in which we need a reliable test: the tall, slender adolescent. The 1996 Ghent criteria were an attempt to condense complex diagnostic thinking into a useful clinical algorithm. I used them regularly and found some shortcomings: they were difficult to use in children, some clinical features were non-specific and unvalidated, and they were written before DNA testing became more widely available. These problems appear to have been addressed in the 2010 update, and the practical problems outlined above should be diminished.

My only slight concern is the emphasis given to the diagnostic category MASS (mitral valve prolapse, aortic root diameter at upper limits of normal [without progression to aneurysm], skin [striae], non-diagnostic skeletal features of Marfan syndrome), although the authors acknowledge some of the difficulties associated with this term. A number of people with possible MFS may be given the diagnosis MASS, but it is unclear how they should be managed, and how many will progress to MFS. Although DNA testing may help, its sensitivity and specificity in this heterogeneous group remains unclear.

\section{REFERENCES}

I Campbell N, Bradshaw N, Davidson R et al. Evidence based medicine in practice: lessons from a Scottish clinical genetics project. J Med Genet 2000; 37:684-9I. doi:I0.I I36/jmg.37.9.684 